ARTICLE

\title{
Atomically-resolved interlayer charge ordering and its interplay with superconductivity in $\mathrm{YBa}_{2} \mathrm{Cu}_{3} \mathrm{O}_{6.81}$
}

Chun-Chih Hsu (10 1, Bo-Chao Huang ${ }^{2}$, Michael Schnedler (1) ${ }^{3}$, Ming-Yu Lai ${ }^{2}$, Yuh-Lin Wang ${ }^{1,2}$, Rafal E. Dunin-Borkowski ${ }^{3}$, Chia-Seng Chang ${ }^{4}$, Ting-Kuo Lee (i] ${ }^{5}$, Philipp Ebert ${ }^{3 凶} \&$ Ya-Ping Chiu (D) ${ }^{1,4,6 凶}$

High-temperature superconductive (SC) cuprates exhibit not only a SC phase, but also competing orders, suppressing superconductivity. Charge order (CO) has been recognized as an important competing order, but its microscopic spatial interplay with SC phase as well as the interlayer coupling in CO and SC phases remain elusive, despite being essential for understanding the physical mechanisms of competing orders and hence superconductivity. Here we report the achievement of direct real-space imaging with atomic-scale resolution of cryogenically cleaved $\mathrm{YBa}_{2} \mathrm{Cu}_{3} \mathrm{O}_{6.81}$ using cross-sectional scanning tunneling microscopy/ spectroscopy. CO nanodomains are found embedded in the SC phase with a proximity-like boundary region characterized by mutual suppression of $\mathrm{CO}$ and superconductivity. Furthermore, SC coherence as well as $\mathrm{CO}$ occur on both $\mathrm{CuO}$ chain and plane layers, revealing carrier transport and density of states mixing between layers. The $\mathrm{CO}$ antiphase correlation along the c direction suggests a dominance of Coulomb repulsion over Josephson tunneling between adjacent layers.

\footnotetext{
${ }^{1}$ Department of Physics, National Taiwan University, Taipei, Taiwan. ${ }^{2}$ Institute of Atomic and Molecular Sciences, Academia Sinica, Taipei, Taiwan. ${ }^{3}$ Peter Grünberg Institut, Forschungszentrum Jülich GmbH, Jülich, Germany. ${ }^{4}$ Institute of Physics, Academia Sinica, Taipei, Taiwan. ${ }^{5}$ Department of Physics, National Sun Yat-sen University, Kaohsiung, Taiwan. ${ }^{6}$ Center of Atomic Initiative for New Materials, National Taiwan University, Taipei, Taiwan.

凶email: p.ebert@fz-juelich.de; ypchiu@phys.ntu.edu.tw
} 
C harge ordering in layered cuprates has been identified as leading competing order to superconductivity and hence as a key factor in shaping and defining the phase diagram ${ }^{1}$. Thus, elucidating the nanostructure of charge order (CO) and its spatial interplay with superconductivity are particularly important for understanding high-temperature superconductivity in cuprates $^{2-11}$. Therefore, CO and its nanostructure have attracted considerable interest for explaining various phenomena: For example, the spatial configuration of $\mathrm{CO}$ has been investigated by several X-ray measurements, which led to the discovery of antiphase interlayer correlations of the charge density wave (CDW) in $\mathrm{YBa}_{2} \mathrm{Cu}_{3} \mathrm{O}_{6+x}\left(\mathrm{YBCO}_{6+x}\right)^{12,13}$ and a three-dimensional (3D) $\mathrm{CDW}$ in thin $\mathrm{YBCO}_{6+x}$ films ${ }^{14}$. The existence of 3D CDWs suggests that charge reservoir layers may act as bridges to transmit a CO correlation along the $\mathrm{c}$ axis of cuprates. However, the exact role of charge reservoir layers in the formation of $\mathrm{CO}$ is still unclear and under debate.

Resolving these critical physical issues and thereby generating a fundamental description of the spatial interplay of $\mathrm{CO}$ and superconductivity in $\mathrm{YBCO}_{6+x}$, requires an atomically resolved real-space characterization of the electronic structure and density-wave correlation layer-by-layer along the c axis. Unfortunately, such results are lacking, despite the prospect to contribute centrally to a fundamental understanding of the competing orders of CDW and superconductivity (SC).

Scanning tunneling microscopy (STM) and spectroscopy (STS) are ideally suited to provide a direct atomically resolved access in real space to electronic properties and thus promises a direct insight into the nature of $\mathrm{CO}$ in $\mathrm{YBCO}_{6+x}$. Prior STM experiments have demonstrated the success of detecting the atomic and electronic structure in real-space on the cleaved c (001) planes ${ }^{15,16}$. Yet, in these measurements, no $\mathrm{CuO}_{2}$ plane can be probed, due to cleavage in between of $\mathrm{BaO}$ or $\mathrm{CuO}$-chain layers ${ }^{15}$. To overcome this restriction, on the one hand, (100) oriented (a) growth surfaces of $\mathrm{YBCO}_{6+x}$ were probed in situ ${ }^{17,18}$, and on the other hand, cross-sectional STM (XSTM) measurements ${ }^{19,20}$ were done on fractured surfaces, albeit without atomic resolution.

In this work, we use a low-temperature cleavage stage to cleave atomically flat cross-sectional surfaces of highly-doped $\mathrm{YBa}_{2} \mathrm{Cu}_{3} \mathrm{O}_{6.81}(p \cong 0.14)^{21}$ layers (room temperature cleavages led to unstable fractures only). The cryogenic cleavage reveals that CO nanodomains are embedded in the SC phase, with a proximity-like boundary region characterized by mutual suppression of $\mathrm{CO}$ and superconductivity. Furthermore, Cooper pair coherence in the SC phase, as well as charge ordering in the $\mathrm{CO}$ phase, occur on both $\mathrm{CuO}$ chain and plane layers, indicating carrier transport and density of states mixing between the layers. The antiphase CO correlation along the $\mathbf{c}$ direction suggests the dominance of Coulomb-interactions between adjacent layers over the Josephson tunneling. Our atomically resolved observations directly reveal the 3D nature of charge order in $\mathrm{YBCO}$, and shed light on the importance of the role played by oxygens for interlayer coupling and complex orders as well as their competition in cuprates $^{22-24}$.

\section{Results}

Due to the bulk structure of orthorhombic YBCO, ideally cleaved (100) surfaces are terminated either by $\mathrm{Ba}-\mathrm{Y}-\mathrm{O}$ or by $\mathrm{Cu}-\mathrm{O}$ layers $^{19}$ (see Fig. 1a). In our XSTM measurement, we indeed observe two types of cleavage surfaces: The former one exhibits a stretched hexagonal-like pattern compatible with a $\mathrm{Ba}-\mathrm{Y}-\mathrm{O}$ terminated surface (see Supplementary Note 1). The latter one, whose STM image is illustrated in Fig. 2a, is characterized by atomic rows perpendicular to the (001) c direction with a periodicity of $0.36 \mathrm{~nm}$. Based on the distinct electronic structure of each layer in $\mathrm{YBCO}$, it can be deduced that the bright rows with higher tunneling conductance arise primarily from copper atoms, which contribute most of the local density of states (LDOS) at the used sample bias of $-50 \mathrm{mV}^{25}$. The dark depressions in between, with lower tunneling conductance, correspond to the insulating layers with barium and yttrium atoms, which do not contribute to the tunnel current significantly. Interestingly, $\mathrm{CuO}$ chain and $\mathrm{CuO}_{2}$ plane layers show an identical contrast in STM images. Figure $2 \mathrm{~b}$ illustrates the Fourier transform image of Fig. 2a. Only a bright peak labeled $\mathbf{Q}_{\text {layer }}$ is resolved, which corresponds to the spacing between the atomic rows (i.e., the $\mathrm{CuO}$ layers) of $0.36 \mathrm{~nm}$.

Furthermore, we acquired tunneling spectra on the $\mathrm{CuO}$ terminated surface on top of the atomic rows (Fig. 2c). Since the crystal structure of YBCO consists of two $\mathrm{CuO}_{2}$ planes separated by one $\mathrm{CuO}$ chain layer along the c direction, we average the spectra acquired on every third atomic row, as marked in Fig. 2a, in order to identify the $\mathrm{CuO}$ chain layers. The spectra of all layers reveal superconductive properties: The differential conductance $(\mathrm{d} I / \mathrm{d} V)$ spectra exhibit a pronounced v-shaped dip structure, which is the signature of a pseudogap, ranging approximately from $-50 \mathrm{mV}$ to $+46 \mathrm{mV}$, in line with the SC critical temperature $T_{\mathrm{C}}=85 \mathrm{~K}$. Superimposed on and thus somewhat hidden in the intense pseudogap slopes one can extract in addition the existence of coherence peaks ${ }^{26}$ and hence a superconductive gap using the negative second derivative of the differential conductance (i.e., $\left.-\mathrm{d}^{2} / \mathrm{d} V^{2}(\mathrm{~d} I / \mathrm{d} V)=-\mathrm{d}^{3} I / \mathrm{d}^{3}\right)$ (inset), which is known to be a sensitive indicator of the position of coherence peak ${ }^{27}$ (since its maxima amplify peaks in the conductance by removing linear backgrounds). Coherence peaks are present in spectra of all layers, suggesting the presence of superconductivity in both $\mathrm{CuO}_{2}$ plane and $\mathrm{CuO}$ chain layers. The SC gap is estimated to be $15 \pm 2$ $\mathrm{mV}$. Note, the apparently weak SC coherence peaks could be related also to the fact that the spectra are acquired in a plane involving the $\mathbf{c}$ axis, along which the SC coherence length is expected to be shorter.

Despite the similarity, a subtle difference can be observed in one of the spectra in Fig. 2c: the blue spectrum exhibits suppressed pseudogap peaks (at negative voltage almost disappearing) as compared to the red and dark red spectra, which are almost identical. Taking into account the crystallography of $\mathrm{YBCO}$, one can assign the blue colored spectrum to the $\mathrm{CuO}$ chain layers, and the red/dark red colored ones to the $\mathrm{CuO}_{2}$ plane layers.

In addition to the dominant SC phase presented above, a minority CO phase occurs in about $15 \%$ of the investigated surface area. Figure 3a displays a STM image of a CO phase, which is characterized by height modulations along the atomic rows at the $\mathrm{Cu}-\mathrm{O}$ terminated surface. The differential conductance image (Fig. 3b) of the same area reveals a charge modulation or CDW as origin of the height modulation. The CDWs are hole density modulations in a hole-doped Mott insulator ${ }^{7}$ and can be considered to be the real space signature of the CO phase. The CDW's wavelength of $1.2 \mathrm{~nm}$ shows up in the Fourier transform (Fig. 3c) as additional (twin) peak (labeled $\left.\mathbf{Q}_{\mathrm{CDW}}\right)$. The position of the atomic row-induced peak $\mathbf{Q}_{\text {layer }}$ remains fixed, corroborating the interpretation that the COinduced modulation is superimposed on the contrast of the atomic rows. The appearance of $\mathbf{Q}_{\mathrm{CDW}}$ twin peaks is related to the anti-phase correlation of $\mathrm{CO}$ along the $\mathbf{c}$ direction discussed below.

Furthermore, Fig. $3 \mathrm{~d}$ shows the averaged $\mathrm{d} I / \mathrm{d} V$ spectra measured on three consecutive layers in the CO area (green lines). For comparison an average $\mathrm{d} I / \mathrm{d} V$ spectrum of the SC phase area is shown as red line (average of spectra in Fig. 2c). Four distinct features can be recognized: (i) no significant difference is observed between $\mathrm{CuO}$ chain and $\mathrm{CuO}_{2}$ plane layers in $\mathrm{CO}$ area. 
a LT-UHV cleavage

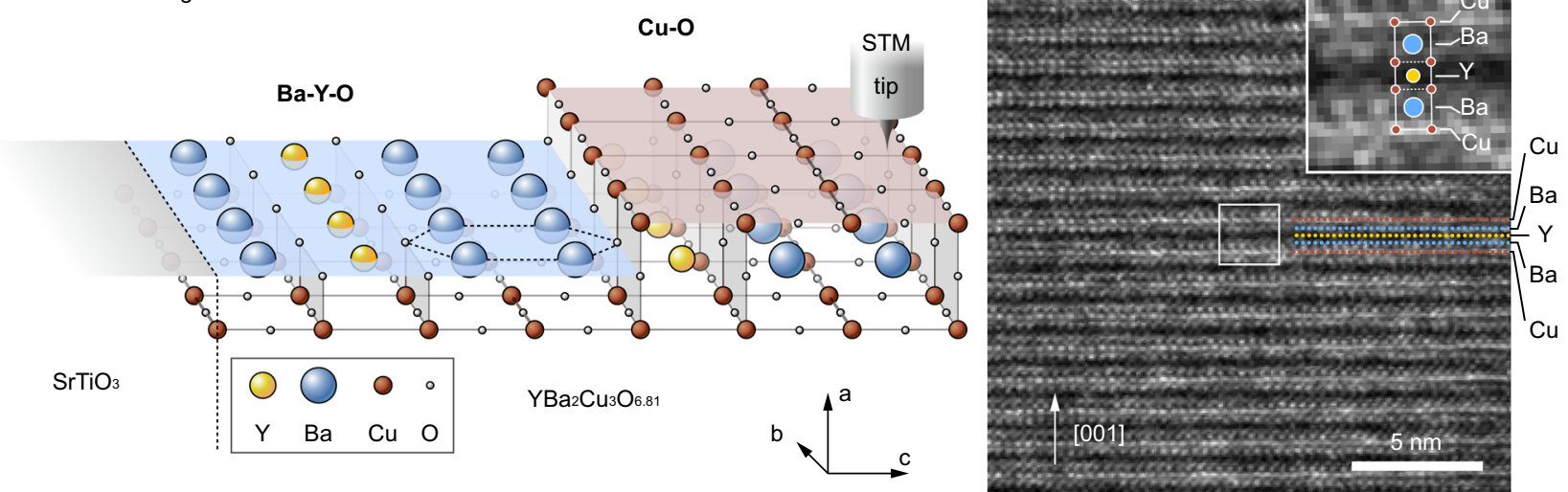

Fig. 1 YBCO cleavage surfaces and layered structure. a Schematic illustration of cross-sectional STM measurements on the $\mathrm{YBa}_{2} \mathrm{Cu}_{3} \mathrm{O}_{6.81} / \mathrm{SrTiO}_{3}$ cleavage surfaces, which are either $\mathrm{Ba}-\mathrm{Y}-\mathrm{O}$ or $\mathrm{Cu}-\mathrm{O}$ terminated. b Bright field cross-sectional TEM image (200 keV) taken $50 \mathrm{~nm}$ away from the $\mathrm{YBa}_{2} \mathrm{Cu}_{3} \mathrm{O}_{6.81} / \mathrm{SrTiO}_{3}$ interface revealing the epitaxial $\mathrm{YBCO}$ layers. The inset shows a magnification with an indicated unit cell.
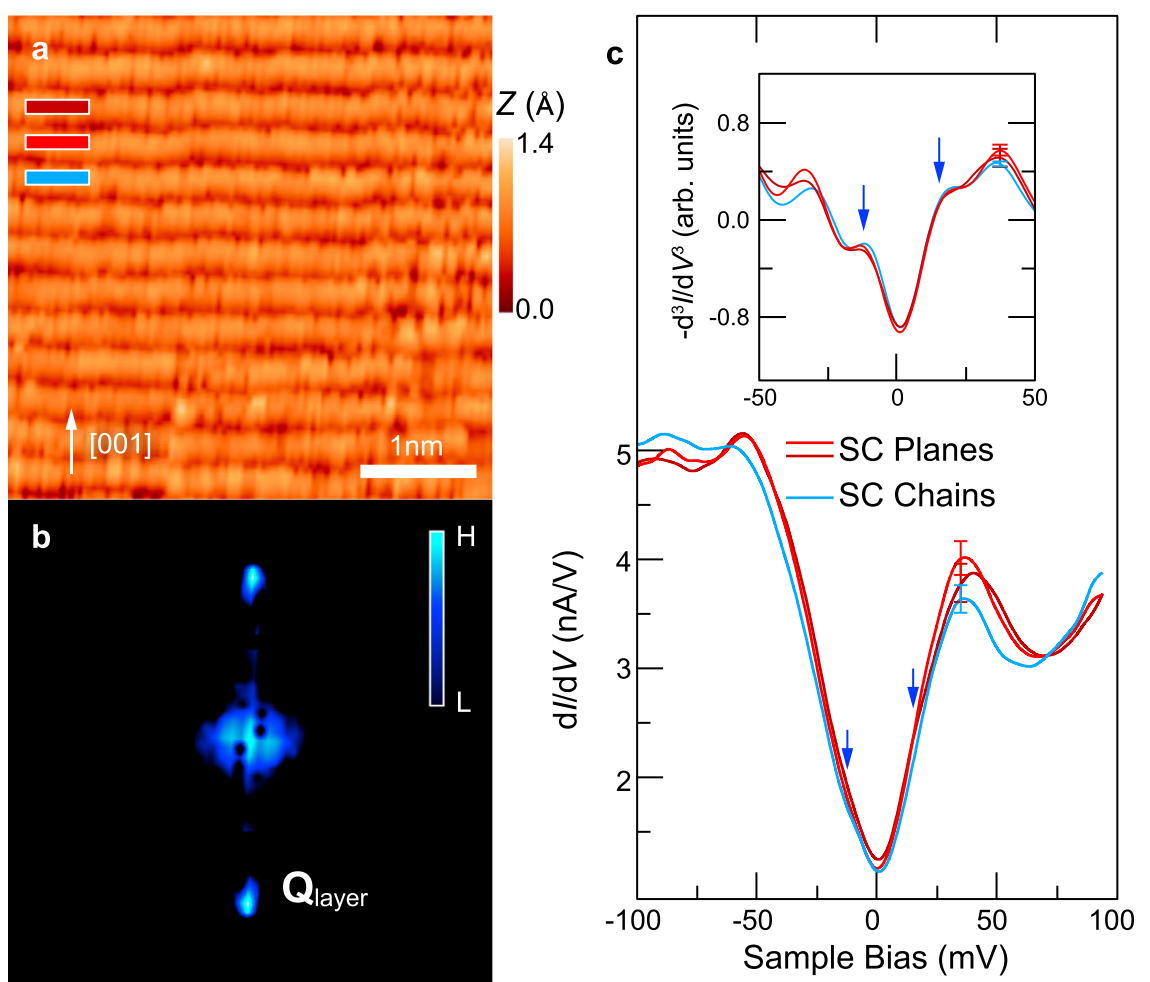

Fig. 2 Superconducting region on $\mathbf{C u}-\mathbf{O}$ terminated surface. a Constant-current STM image of the $\mathrm{Cu}-\mathrm{O}$ terminated YBCO surface measured at a sample bias of $-50 \mathrm{meV}$ and a current of $200 \mathrm{pA}$. The STM image exhibits a clear layered structure perpendicular to [001] direction, corresponding to tunneling into states associated with the $\mathrm{CuO}$-chain and $\mathrm{CuO}_{2}$-plane layers. b Fourier transform of the STM image in (a). The peaks labeled $\mathbf{Q}_{\text {layer }}$ arise from the imaged atomic planes. c Differential conductance spectra obtained from three consecutive atomic layers corresponding to $\mathrm{CuO}_{\text {-chain }}$ and $\mathrm{CuO}_{2}$-plane layers. The blue arrow marks the superconductive (SC) gap as a weak bulge on the flanks of the pseudogap. The inset displays the negative second derivative of the differential conductance spectra, $-d^{3} I / d V^{3}$, measured from the three consecutive layers, revealing the coherence peaks of the SC gap and providing the signature to identify the SC phase. A representative error bar is given for each spectrum.

(ii) The $\mathrm{d} I / \mathrm{d} V$ curves in the CO domain reveal strongly suppressed pseudogap peaks (at $-50 \mathrm{meV}$ and $+46 \mathrm{meV}$ ) as well as no local dip at $+65 \mathrm{meV}$. (iii) The $-\mathrm{d}^{3} I / \mathrm{d} V^{3}$ spectra reveal no SC coherence peaks. (iv) Finally, a clear upward shift by $7 \mathrm{meV}$ of the pseudogap peak and the gap's minimum occurs, as compared to the SC region. These features demonstrate that the CO phase is distinctively different than the SC phase. In particular, we note that no CO can be detected in the SC phase as supported by the different electronic properties and the absence of any modulation in constant current as well as conductivity maps (Fig. 2a and
Supplementary Fig. 2). Note, these results rule out quasiparticle scattering as origin of the observed modulations, since quasiparticle scattering should only occur in presence of $\mathrm{SC}^{11}$. Hence, the dominant contributions to the modulations observed in STM can be assigned to CDWs.

The most intriguing LDOS feature in the CO domain is the upward shift of the pseudogap minimum, which suggests a particle-hole asymmetric gap. The shift creates a significant increase of the density of states (DOS) directly at the Fermi level $\left(E_{\mathrm{F}}\right)$, which is in conflict with a strongly reduced single-particle 


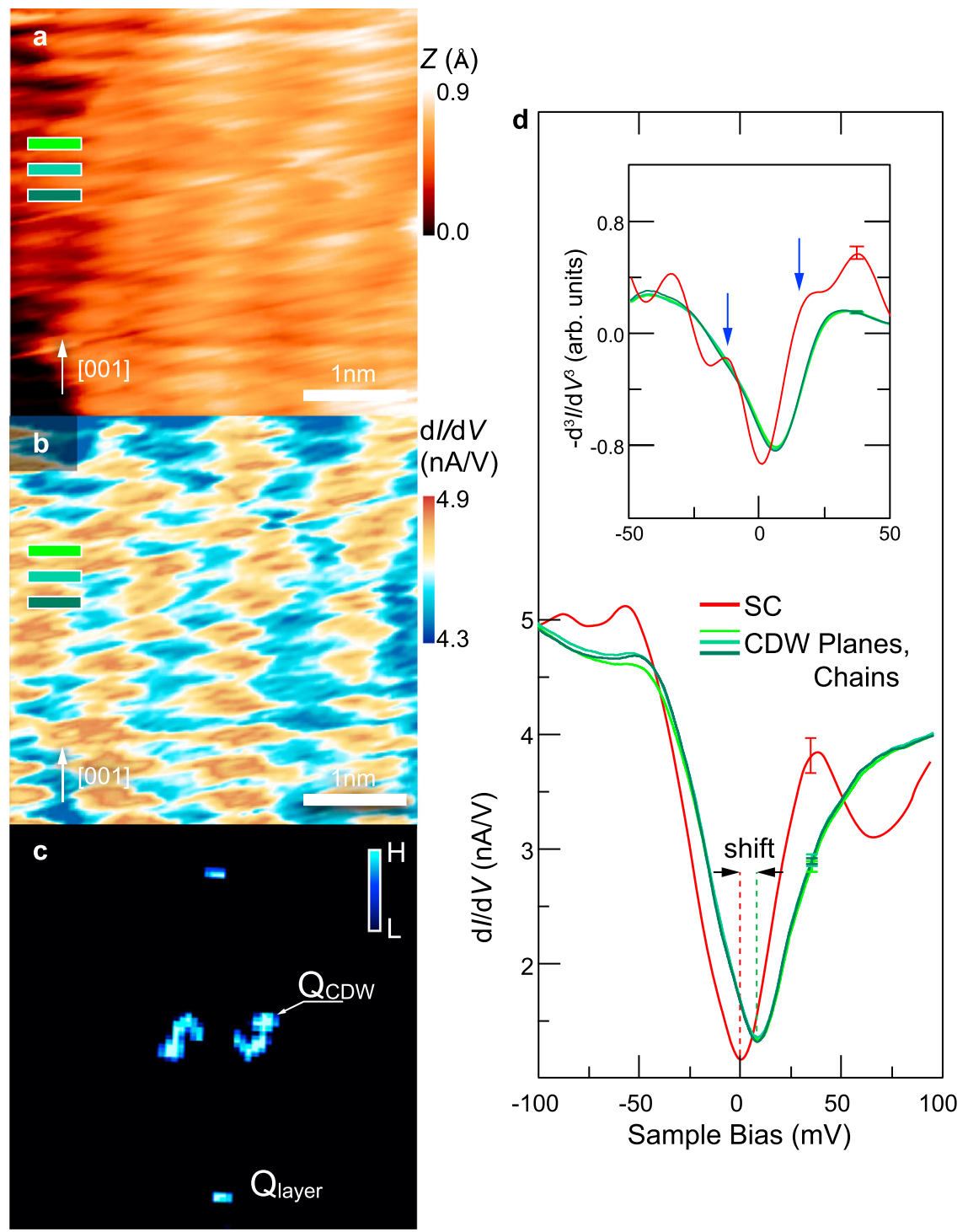

Fig. 3 Charge density wave (CDW) region on Cu-O terminated surface. a Topographic image obtained with a sample bias of $+50 \mathrm{mV}$ and $200 \mathrm{pA}$, showing the presence of the charge modulation along the $\mathbf{b}$-axis. $\mathbf{b}$ The corresponding differential tunneling conductance map at $+50 \mathrm{mV}$ sample bias. c Fourier transformation of the image in (b). d Differential tunneling spectra in CDW region obtained with a setpoint of $-50 \mathrm{mV}$ and $200 \mathrm{pA}$. The red spectrum shows the average LDOS in SC-phase for comparison. Spectra colored in different shades of green correspond to $\mathrm{d} / \mathrm{d} V \mathrm{taken}$ from three consecutive layers. The inset shows $-d^{3} l / d V^{3}$, in which the superconducting gap is marked by the blue arrows. A representative error bar is given for each spectrum.

DOS at $E_{\mathrm{F}}$ in the SC phase. Hence, we anticipate that the shift of the minimum of single-particle DOS away from $E_{\mathrm{F}}$ is intimately interrelated with and sets the basis for $\mathrm{CO}^{28,29}$. As side effect, the upward shift can be anticipated to result in reducing the Cooper pair bonding energy and hence the Cooper pair concentration and coherence, thereby suppressing superconductivity.

Furthermore, Fig. 4a and Supplementary Fig. 3 illustrates that nanoscale $\mathrm{CO}$ domains ${ }^{30,31}$ are coexisting with and inserted in between of SC areas. Figure 4b illustrates the average tunneling spectrum measured at the boundary. It exhibits features, which distinguish it from both the spectra measured in the center of the CO domain and in the SC phase: first, the minimum of the DOS dip is again at the Fermi energy and a pseudogap is formed, although with weakly developed edge peaks. Second, no superconductive energy gap and coherence peaks can be detected. Hence, a mutual suppression of $\mathrm{CO}$ and SC occurs in a proximity-like boundary region.
After the electronic characteristics of CO and SC phases as well as their boundary, we address the real space pattern of the CDWs in Figs. 5 and 6. Since it is known that the intensity of the wave vector and thus the real space modulation intensity of the CDW reaches a maximum at the pseudogap energy ${ }^{11}$ (i.e., $\pm 50 \mathrm{meV}$ ) we chose these voltages for mapping the modulations of the CO. Figure $5 \mathrm{a}$ and $\mathrm{b}$ illustrate a pair of $\mathrm{d} I / \mathrm{d} V$ maps probed at +50 and $-50 \mathrm{mV}$, revealing $\mathrm{CDW}$-induced modulations. The two wave patterns are not identical, suggesting a voltage dependence of the charge modulation ${ }^{16,32}$. For a deeper understanding of the CDW, we evaluate its wavelength $\lambda$ (i.e., the peak-to-peak distance between two modulation maxima as defined in Fig. 6a) for every atomic row (i.e., chain and plane layers) in all of the sampled CO region. Figure $6 \mathrm{~b}$ shows the obtained distributions of $\lambda$ at bias voltages of +50 and $-50 \mathrm{mV}$, respectively. Both distributions exhibit two peaks centered around 1.2 and $1.6 \mathrm{~nm}$ wavelength. The data suggest that the dominating wavelength shifts from 1.2 

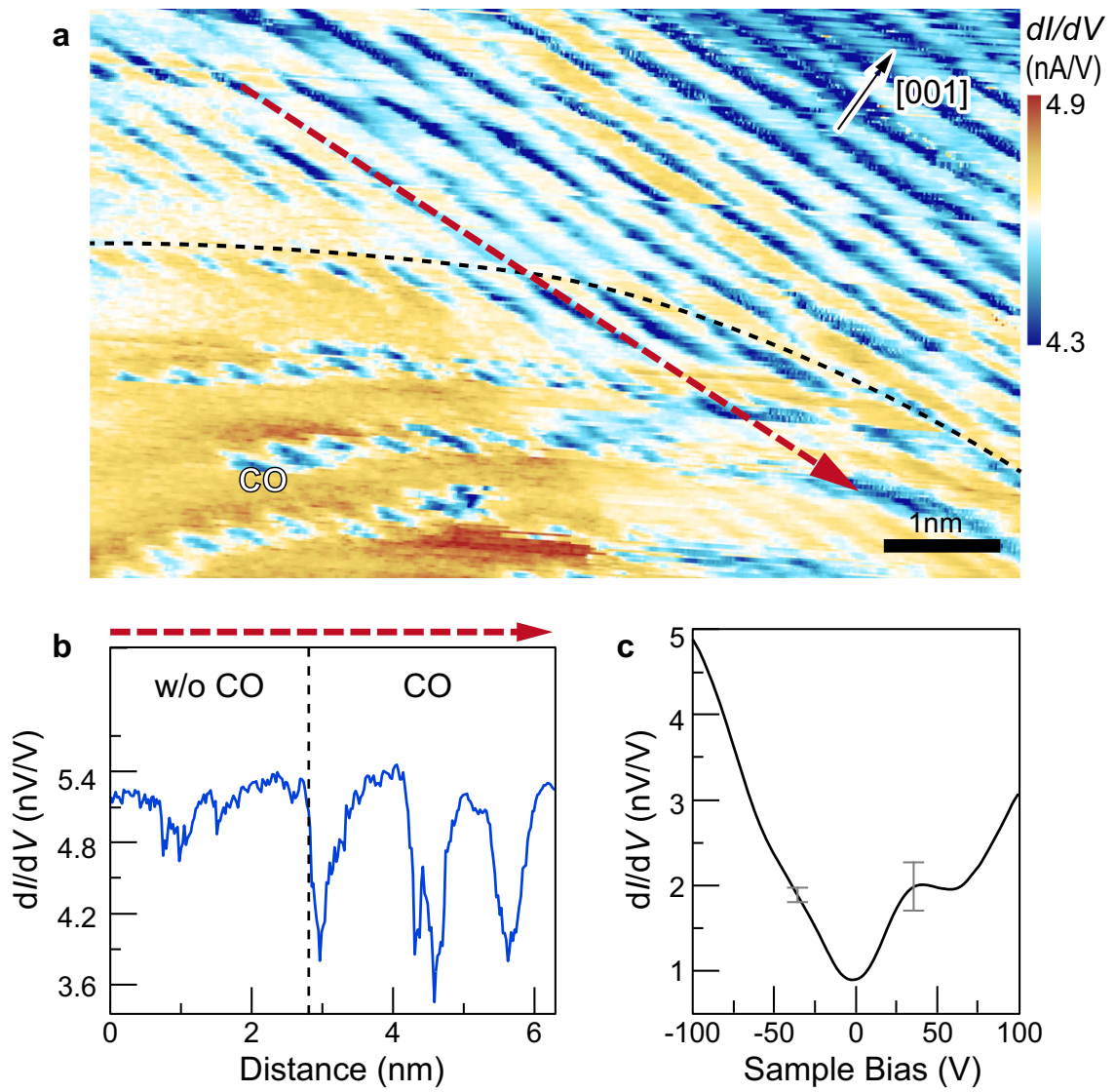

Fig. 4 The CO phase boundary and STS at the boundary. a Differential conductance map of the CO phase boundary measured at a sample bias of +200 $\mathrm{mV}$ and tunneling current of $500 \mathrm{pA}$. The dashed line indicates the approximate position of the CO phase boundary. $\mathbf{b}$ We used line profiles along the atomic rows in $\mathbf{b}$ direction to define the $\mathrm{CO}$ phase boundary. The $\mathrm{CO}$ phase shows up as a wavy corrugation (height change), which suddenly terminates. The spatial position where the modulation disappears and the contrast becomes constant is taken as boundary. $\mathbf{c}$ Average tunneling spectrum measured at the $\mathrm{CO}$ phase boundary with two representative error bars given at positive and negative bias.
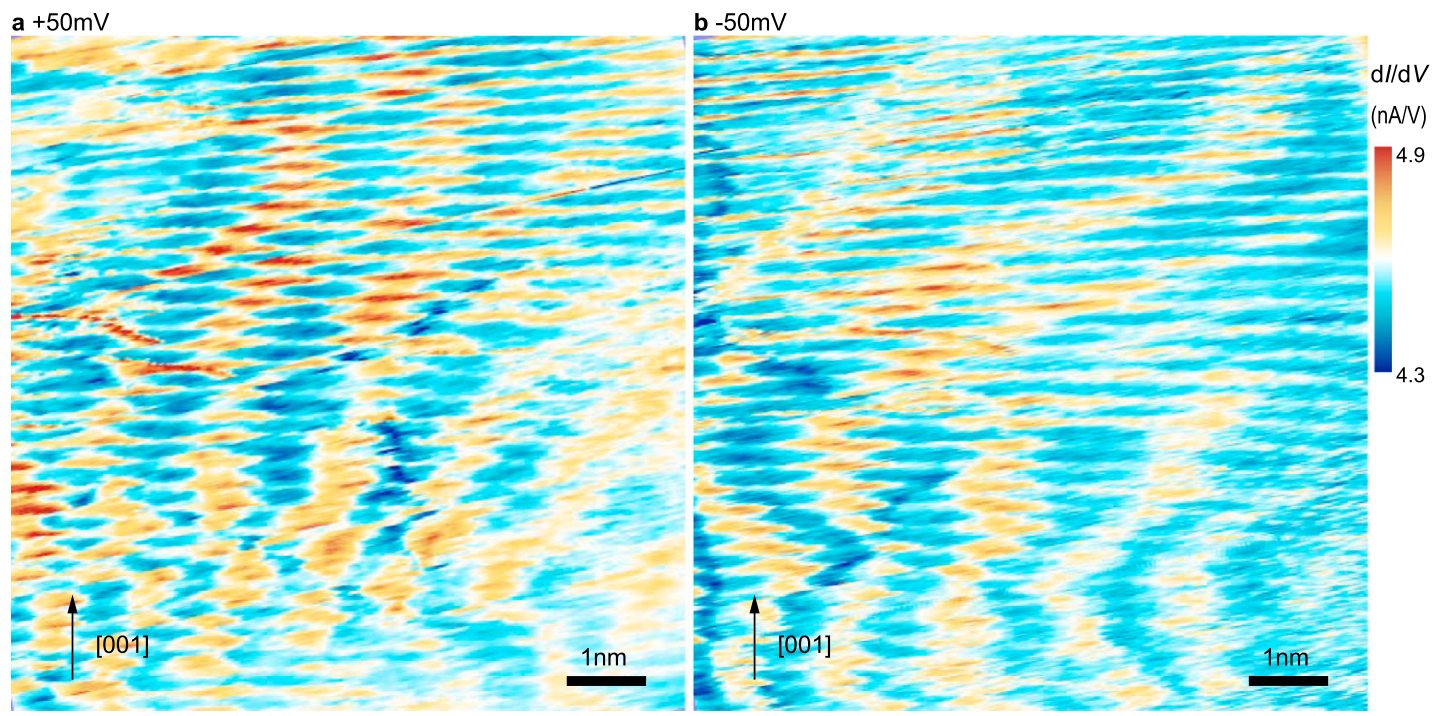

Fig. 5 Dependency of CDW pattern on the polarity of sample bias. a, $\mathbf{b}$ Conductance map of a surface area within a CO domain measured at sample bias of $\mathbf{a}+50 \mathrm{mV}$ and $\mathbf{b}-50 \mathrm{mV}$. The tunnel current is $200 \mathrm{pA}$.

$\mathrm{nm}$ at $+50 \mathrm{mV}$ to $1.6 \mathrm{~nm}$ at $-50 \mathrm{mV}$. Furthermore, one can discern a general curvy pattern of the CDW maxima near the edges of the nanoscale $\mathrm{CO}$ domains (Fig. 4a), where the longer wavelength is preferentially present. For understanding the distribution of wavelength, we recall that the periodicity of $\mathrm{CO}$ depends on the doping level of $\mathrm{YBCO}^{2}$. Thus, for a given doping level, the periodicity of $\mathrm{CO}$ is an intrinsic property. The wavelength of $\lambda=1.2 \mathrm{~nm}$ is consistent with the position of the CDW's Fourier peak in Fig. $3 \mathrm{c}$ and with the wave vectors of CDWs measured by X-ray experiments ${ }^{2,9}$, coinciding with but not 

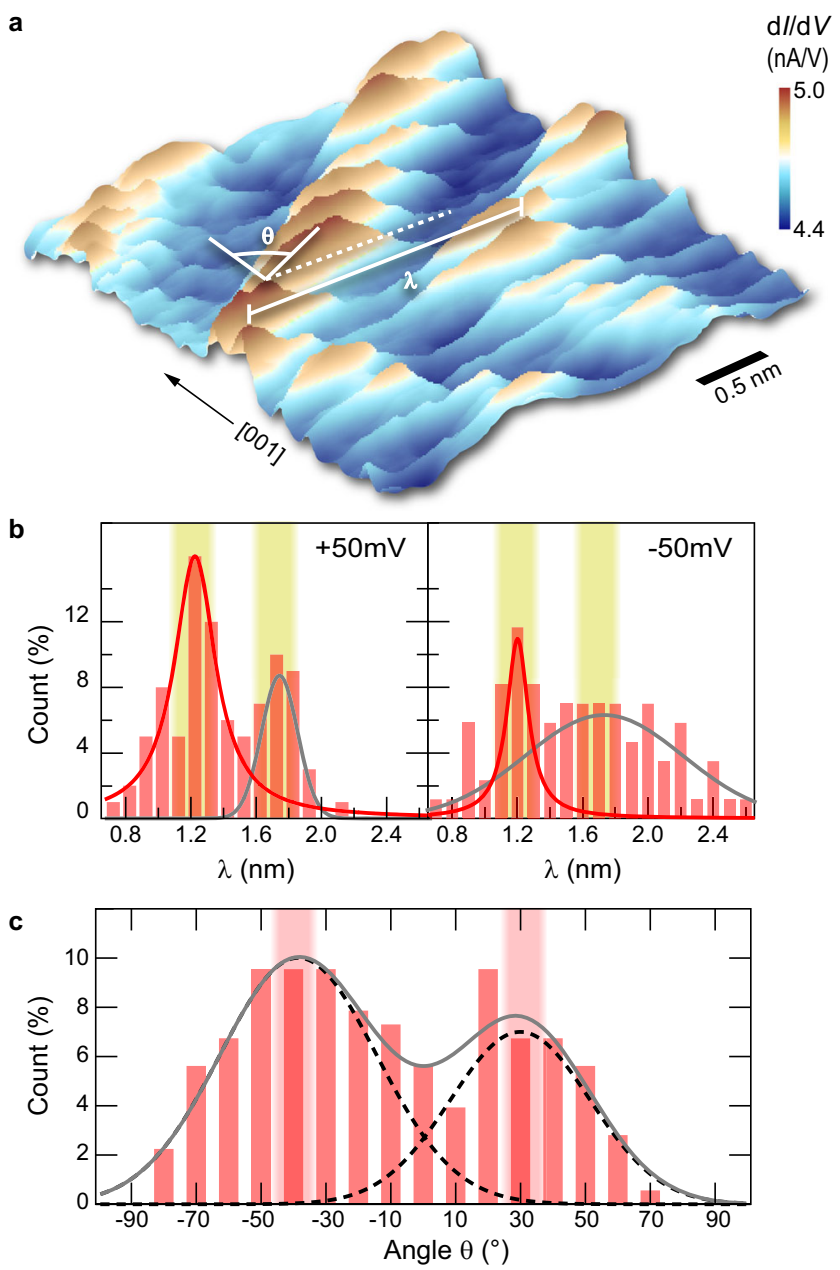

d

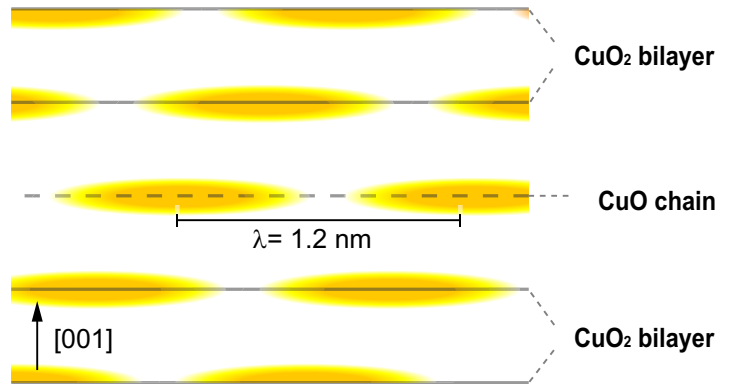

Fig. 6 Wavelength and angular distribution of CDW. a Three-dimensional representation of a $\mathrm{d} / / \mathrm{d} V$ image showing charge modulations on the atomic rows in a false-color scale (acquired at a tunnel voltage of $+50 \mathrm{mV}$ ). Indicated are the definitions used to measure the wavelength $\lambda$ and the angle $\theta$ describing the spatial correlation of charge modulations in adjacent atomic rows. $\mathbf{b}$ Histograms of the distribution of wavelength $\lambda$ measured at (left) $+50 \mathrm{mV}$ and (right) $-50 \mathrm{mV}$ tunneling voltage. One can distinguish a peak at $\lambda=1.2 \mathrm{~nm}$ (red lines: Lorentzian fits) and another peak at $\sim \lambda=1.6$ $\mathrm{nm}$ (gray lines are Gaussian fits) for both tunneling voltages. c Frequency distribution of the angle $\theta$ describing the correlation of the charge modulation across the atomic rows in $\mathbf{c}$ ([001]) direction. Angles $\theta$ can be best described with two Gaussians, centered around $-40^{\circ}$ and $+30^{\circ}$. The presence of two shifted peaks indicate a short-range anti-correlation ordering of parallel CDW stripes along the [001] direction, as schematically illustrated in (d). necessarily originating from the oxygen-site occupation induced periodicity of chain superlattice in ortho-III ordered $\mathrm{YBCO}^{33}$. The presence of longer wavelengths can be explained on basis of fluctuations of the local oxygen site occupation, especially near the $\mathrm{CO}$ phase boundary. Note, due to the possible influences of the tip's electric field on the sample and the curvy pattern especially near the edges of the CO phase areas, one cannot derive an energy-dependent dispersion from the measured voltage dependence.

Besides the presence of charge-order modulation on single layers, the STM images indicates a correlation of the CDWs along the perpendicular $\mathbf{c}([001])$ direction. In order to support the correlation, we evaluate the angle $\theta$ defined in Fig. $6 \mathrm{a}$ as the angle between the $\mathbf{c}$ direction and the stripe-like CDW pattern. Figure $6 \mathrm{c}$ illustrates the obtained distribution of occurrences of angles $\theta$, which can be best described with two Gaussians, centered around $-40^{\circ}$ and $+30^{\circ}$. Near $0^{\circ}$ a minimum occurs. The distribution significantly deviates from that expected for uncorrelated CDWs (random distribution) or for in phase $\mathrm{CO}$ correlation in [001] direction (single peaks centered at $0^{\circ}$ ). The presence of two shifted peaks indicates a phase-shifted interlayer $\mathrm{CO}$ correlation in [001] direction, i.e., a short-range anti-correlation ordering of parallel CDW stripes along the [001] direction, as schematically illustrated in Fig. $6 \mathrm{~d}$.

\section{Discussion}

The above results raise several points, which require an in-depth discussion: (i) the relation of $\mathrm{CO}$ and SC phase, (ii) the layer resolved SC properties, (iii) the layer resolved $\mathrm{CO}$, and (iv) the anti-phase $\mathrm{CO}$ correlation across the layers.

First, the observation of CO domain embedded in the SC phase can be rationalized as follows: The periodicity of the $\mathrm{CO}$ is known to depend directly on the oxygen content (i.e., hole concentration $\left.{ }^{2,34}\right)$. This suggests that the samples exhibit local fluctuations of the hole concentration ${ }^{30}$ in analogy to dopant fluctuations in semiconductors ${ }^{35}$. These fluctuations can be expected to induce local CO domains with hole density changes toward the edges of the domain, giving rise to curvy CO pattern. Hence, the formation of $\mathrm{CO}$ nanoscale domains can be understood in terms of quenched disorder and local inhomogeneity in cuprate superconductors.

With this in mind, we anticipate that the electronic features at the CO-SC-phase boundary reflect a reduced free carrier concentration at $E_{\mathrm{F}}$ as compared to that in the center of the CO domain. This reduced carrier density suppresses $\mathrm{CO}$ and allows Cooper pairs to form, which can be inferred from the reformation of a pseudogap with edge peaks. However, no SC coherence peaks are detected in the boundary region. One could call this a proximity effect between $\mathrm{CO}$ and SC phases, in analogy to SCmetal contacts. This suggests that $\mathrm{CO}$ and SC are indeed competing and immiscible orders. This is corroborated by the fact that although both phases occur in the same sample, the respective electronic features and order parameters (SC coherence peaks and shift of DOS minimum) do not overlap and thus the phases are not found to intermix. The tunneling spectra suggest that the competition is likely defined by the competition for the charge carriers, as both phases need carriers, one for a superconducting state, and the other as free single carriers which undergo a CO. Note, at present there is not experimental data on how wide the boundary region is. Furthermore, defects in the YBCO stacking order should not be responsible for the formation of CO phase embedded in SC phase, because the size of areas free of stacking defects is significantly larger than the observed size of the $\mathrm{CO}$ areas. 
Second, in the SC phase, we observed that the $\mathrm{d} I / \mathrm{d} V$ curves of the $\mathrm{CuO}$ chain and $\mathrm{CuO}_{2}$ plane layers exhibits the same overall shape including a superconducting gap and the corresponding coherence peaks (Fig. 2). This suggests that both, the chain and plane layers, exhibit superconductive properties, in line with previous observations of a SC gap in the chain layer ${ }^{36,37}$. This and the observation of a SC gap in tunneling spectra of the Ba-Y-O terminated surface (see Supplementary Fig. 1) indicate that electrons can tunnel/hop from layer to layer in $\mathrm{YBCO}$, due to the small layer separation of about $0.4 \mathrm{~nm}$. Similarly, we anticipate that Cooper pairs are able to cross insulating layers via Josephson tunneling. In addition, one can speculate that the oxygen atoms in insulating $\mathrm{Ba}-\mathrm{O}$ layers and their bonds provide a channel for carriers and Cooper pairs to connect neighboring $\mathrm{CuO}$ chain/ plane layers. As a consequence, one would expect to observe the same single-particle DOS on both chain and plane layers in terms of SC gap and coherence peaks, decaying along the $\mathbf{c}$ direction, but with significant overlap between adjacent $\mathrm{Cu}-\mathrm{O}$ layer. This picture of carrier and Cooper pair hopping/tunneling is directly supported by our measurements (see also Supplementary Note 4) and could be related to previously suggested possible intermixing of the LDOS between $\mathrm{CuO}$ chain and $\mathrm{CuO}_{2}$ plane layers ${ }^{36,37}$.

Third, we address the presence of $\mathrm{CO}$ in $\mathrm{CuO}$ chain and plane layers and its correlation across the layers. These findings confirm again the possibility of carrier hopping/tunneling between the $\mathrm{Cu}-\mathrm{O}$ layers and indicate an interaction of the $\mathrm{CO}$ across the $\mathrm{CuO}$ layers. The presence of an interaction of $\mathrm{CO}$ across the $\mathrm{CuO}$ layers can be also inferred from a prior observed transition of a $3 \mathrm{D}$-like to a $2 \mathrm{D}$-like $\mathrm{CO}$ behavior with decreasing doping, i.e., increasing oxygen vacancy concentration ${ }^{14}$. In the highly underdoped YBCO with unoccupied oxygen sites in the $\mathrm{CuO}$ chain layers, the copper atoms (in the chain layer) have essentially a fully occupied $3 d^{10}$ electron configuration. As the oxygen content increases, the vacancy sites in the chain are occupied by oxygen and the coppers' electron configuration evolves from $3 d^{10}$ toward $3 d^{9}$. This results in holes located at the oxygen atoms in the $\mathrm{CuO}$ chain. Hence, for strongly underdoped $\mathrm{YBCO}$, the $\mathrm{Cu}$ atoms in the chain layer exhibit a fully occupied $3 d$ shell, which one can anticipate to block the electron pathway between the adjacent layers in $\mathbf{c}$ direction resulting in a $2 \mathrm{D}$-like behavior. In contrast, in optimally doped $\mathrm{YBCO}$, the $\mathrm{Cu}$ atoms in the chain layer have an incompletely filled $3 d$ shell, which should allow electron hopping between the layers, resulting in a 3D-like electronic behavior. The presence of such conduction pathways is further corroborated by an electronic rehybridization of the $\mathrm{Cu}$ (chain)-apical O(4)-Cu(plane)-orbitals ${ }^{23}$.

Finally, we turn to the anti-phase correlation of $\mathrm{CO}$ across the layers (showing up in the angle distribution in Fig. $6 \mathrm{c}$ and schematically illustrated in Fig. 6d). The $\mathrm{CO}$ correlation across the layers has been suggested to be governed by Josephson tunneling as well as charge interaction between the layers ${ }^{38}$. Since Josephson tunneling favors an in-phase correlation, our experimental results suggest that the antiphase pattern observed here is dominated by the minimization of Coulomb interaction energy between layers ${ }^{5,38}$. As further support for the 3D CO structure, we recall that on the $\mathrm{Ba}-\mathrm{Y}-\mathrm{O}$ terminated surface we never observed any charge density modulation, suggesting that indeed no $\mathrm{CO}$ occurs on the $\mathrm{Ba}-\mathrm{Y}-\mathrm{O}$ layer. This corroborates the identification of the $\mathrm{CO}$ consisting of one-dimensional stripes along the $\mathbf{b}$ direction on the $\mathrm{Cu}-\mathrm{O}$ planes with anticorrelation in $\mathbf{c}$ direction.

We revealed the interlayer coupling and the spatial interplay and competition of the charge ordered and the superconductive phases in $\mathrm{YBa}_{2} \mathrm{Cu}_{3} \mathrm{O}_{6.81}$ by direct real-space imaging with atomicscale resolution using cross-sectional scanning tunneling microscopy and spectroscopy on cryogenic cleavage surfaces. We found that highly-doped $\mathrm{YBCO}$ with high $T_{\mathrm{C}}$ contains nanosized 3D- correlated CO domains embedded in the SC phase, and attribute this to fluctuations of the local doping. $\mathrm{CO}$ is found to be antiphase correlated in $\mathbf{c}$ direction suggesting a dominance of Coulomb-repulsion over Josephson-tunneling. In the SC phase the tunneling spectra reveal the presence of a SC energy gap and coherence peaks on $\mathrm{CuO}$ chain as well as plane layers. In the $\mathrm{CO}$ phase, an upward shift of the minimum of the pseudogap, attributed to an asymmetric particle hole gap, and no coherence peaks were found on both $\mathrm{CuO}$ chain and plane layers, too. The presence of almost identical LDOS on chain and plane layers in both phases is believed to be a manifestation of carrier transport across the $\mathrm{CuO}$ layers and indicative of a density of states mixing, anticipated to be driven by the rehybridization of apical oxygen atoms with the neighboring $\mathrm{Cu}$ atoms in the chain and plane layers. At the CO-SC phase boundary, the tunneling spectra reveal a proximity-like zone, where neither SC gap nor the upward DOS shift characteristic of the CO phase, can be detected, suggesting a mutual suppression of $\mathrm{CO}$ and superconductivity. We anticipate that both phases are competing for the same carriers and are thus immiscible orders. The results revealed by cross-sectional STM/STS provide direct evidence of the 3D nanostructure and spatial interplay of competing, immiscible orders with superconductivity and a fundamental insight into the role of apical oxygen atoms for interlayer coupling and understanding complex competing orders in cuprates.

\section{Methods}

For the experiments we investigated a $400 \mathrm{~nm}$ thick (001) oriented $\mathrm{YBCO}_{6.81}$ (YBCO) thin film, grown on a $\mathrm{SrTiO}_{3}$ (STO) with (001) oriented substrate using pulsed laser sputter deposition. Pieces cut from the sample were cleaved on crosssectional a (100) planes at $10 \mathrm{~K}$ in-situ in the ultrahigh vacuum (UHV) chamber Immediately after cleavage the samples were investigated at $4.3 \mathrm{~K}$ by STM/STS without interruption of the vacuum after cleavage. Figure la shows a schematic illustration of the bulk-like cleavage planes and the used low-temperature XSTM setup. The low-temperature (LT) cleavage and measurement avoids changes of the oxygen content under UHV conditions, essential for probing intrinsic properties of YBCO on bulk-like cleavage surfaces. Furthermore, in order to explore unstrained YBCO, we probed a region about $150 \mathrm{~nm}$ away from the YBCO/STO interface, where the stress from the lattice mismatch is generally released. Finally, throughout the YBCO film we observed a well-ordered $\mathbf{c}$ axis oriented atomic structure, as illustrated by the bright field transmission electron microscopy (TEM) image in Fig. 1b. The YBCO had a high critical temperature of $T_{\mathrm{C}} \cong 85 \mathrm{~K}$.

STS measurements were carried out using the lock-in technique (bias modulation $\delta V=2 \mathrm{mV}, f=708 \mathrm{~Hz}$ ). Using this lock-in technique, standard $\mathrm{d} I / \mathrm{d} V$ vs. $V$ spectra as, well as topographic differential conductance maps $\mathrm{d} I / \mathrm{d} V\left(\mathbf{r}, V_{\text {set }}\right) \equiv \mathrm{g}(\mathbf{r}$, $V_{\text {set }}$ ) for a fixed set voltage $V_{\text {set }}$, were acquired. The latter is used to map across the surface the LDOS information at the energy corresponding to the set voltage.

For obtaining a tunneling spectrum at a selected pixel point, i.e., spatial positions, five $\mathrm{d} I / \mathrm{d} V$ spectra were measured consecutively and averaged. Then, these tunneling spectra were further averaged for spatially equivalent pixel positions, e.g., along the same type of atomic plane/row. Finally, a running average with a width of $3 \mathrm{mV}$ was applied for smoothing, resulting in the averaged spectra shown in Figs. 2c, 3d, and 4c.

\section{Data availability}

The data that support the findings of this study are available from the corresponding author upon reasonable request.

Received: 3 October 2020; Accepted: 31 May 2021; Published online: 23 June 2021

\section{References}

1. Fradkin, E., Kivelson, S. A. \& Tranquada, J. M. Colloquium: theory of intertwined orders in high temperature superconductors. Rev. Mod. Phys. 87, 457-482 (2015).

2. Hücker, M. et al. Competing charge, spin, and superconducting orders in underdoped $\mathrm{YBa}_{2} \mathrm{Cu}_{3} \mathrm{O}_{y}$. Phys. Rev. B 90, 054514 (2014).

3. Hoffman, J. E. et al. A four unit cell periodic pattern of quasi-particle states surrounding vortex cores in $\mathrm{Bi}_{2} \mathrm{Sr}_{2} \mathrm{CaCu}_{2} \mathrm{O}_{8+\delta}$. Science 295, 466-469 (2002). 
4. Howald, C., Eisaki, H., Kaneko, N., Greven, M. \& Kapitulnik, A. Periodic density-of-states modulations in superconducting $\mathrm{Bi}_{2} \mathrm{Sr}_{2} \mathrm{CaCu}_{2} \mathrm{O}_{8+\delta}$. Phys Rev. B 67, 014533 (2003).

5. Chang, J. et al. Direct observation of competition between superconductivity and charge density wave order in $\mathrm{YBa}_{2} \mathrm{Cu}_{3} \mathrm{O}_{6.67}$. Nat. Phys. 8, 871-876 (2012).

6. Comin, R. et al. Charge order driven by Fermi-arc instability in $\mathrm{Bi}_{2} \mathrm{Sr}_{2-x} \mathrm{La}_{x} \mathrm{CuO}_{6+\delta}$. Science 343, 390-392 (2014).

7. Da Silva Neto, E. H. et al. Ubiquitous interplay between charge ordering and high-temperature superconductivity in cuprates. Science 343, 393-396 (2014).

8. Ghiringhelli, G. et al. Long-range incommensurate charge fluctuations in (Y,Nd) $\mathrm{Ba}_{2} \mathrm{Cu}_{3} \mathrm{O}_{6+x}$. Science 337, 821-825 (2012).

9. Achkar, A. J. et al. Distinct charge orders in the planes and chains of ortho-IIIordered $\mathrm{YBa}_{2} \mathrm{Cu}_{3} \mathrm{O}_{6+\delta}$ superconductors identified by resonant elastic X-ray scattering. Phys. Rev. Lett. 109, 167001 (2012).

10. Blackburn, E. et al. X-ray diffraction observations of a charge-density-wave order in super- conducting ortho-II $\mathrm{YBa}_{2} \mathrm{Cu}_{3} \mathrm{O}_{6.54}$ single crystals in zero magnetic field. Phys. Rev. Lett. 110, 137004 (2013).

11. Hamidian, M. H. et al. Atomic-scale electronic structure of the cuprate dsymmetry form factor density wave state. Nat. Phys. 12, 150-156 (2016).

12. Comin, R. et al. Broken translational and rotational symmetry via charge stripe order in underdoped $\mathrm{YBa}_{2} \mathrm{Cu}_{3} \mathrm{O}_{6+y}$. Science 347, 1335-1339 (2015)

13. Forgan, E. M. et al. The microscopic structure of charge density waves in underdoped $\mathrm{YBa}_{2} \mathrm{Cu}_{3} \mathrm{O}_{6.54}$ revealed by X-ray diffraction. Nat. Commun. 6, 10064 (2015)

14. Bluschke, M. et al. Stabilization of three-dimensional charge order in $\mathrm{YBa}_{2} \mathrm{Cu}_{3} \mathrm{O}_{6+x}$ via epitaxial growth. Nat. Commun. 9, 2978-2978 (2018).

15. Edwards, H. L., Markert, J. T. \& de Lozanne, A. L. Energy gap and surface structure of $\mathrm{YBa}_{2} \mathrm{Cu}_{3} \mathrm{O}_{7-x}$ probed by scanning tunneling microscopy. Phys. Rev. Lett. 69, 2967-2970 (1992).

16. Derro, D. J. et al. Nanoscale one-dimensional scattering resonances in the $\mathrm{CuO}$ chains of $\mathrm{YBa}_{2} \mathrm{Cu}_{3} \mathrm{O}_{6+x}$. Phys. Rev. Lett. 88, 097002 (2002).

17. Wei, J. Y. T., Yeh, N.-C., Garrigus, D. F. \& Strasik, M. Directional tunneling and Andreev reflection on $\mathrm{YBa}_{2} \mathrm{Cu}_{3} \mathrm{O}_{7-\delta}$ single crystals: predominance of $d$ -wave pairing symmetry verified with the generalized Blonder, Tinkham, and Klapwijk theory. Phys. Rev. Lett. 81, 2542-2545 (1998).

18. Yeh, N.-C. et al. Evidence of doping-dependent pairing symmetry in cuprate superconductors. Phys. Rev. Lett. 87, 087003 (2001).

19. Chen, C. J., Tsuei, C. C., Watson, T. J. \& Heights, Y. Topography and local conductance images of $\mathrm{YBa}_{2} \mathrm{Cu}_{3} \mathrm{O}_{7}$ crystals fractured in ultra high vacuum. Solid State Commun. 71, 33 (1989)

20. Ren, M.-Q., Z., T., Yan, Y.-J. \& D-L, F. Possible nodeless superconducting gaps in $\mathrm{Bi}_{2} \mathrm{Sr}_{2} \mathrm{CaCu}_{2} \mathrm{O}_{8+\delta}$ and $\mathrm{YBa}_{2} \mathrm{Cu}_{3} \mathrm{O}_{7-x}$ revealed by cross-sectional scanning tunneling spectroscopy. Chin. Phys. Lett. 33, 127402 (2016).

21. Liang, R., Bonn, D. A. \& Hardy, W. N. Evaluation of $\mathrm{CuO}_{2}$ plane hole doping in $\mathrm{YBa}_{2} \mathrm{Cu}_{3} \mathrm{O}_{6+x}$ single crystals. Phys. Rev. B 73, 180505 (2006)

22. Hartman, M. et al. Direct observation of apical oxygen vacancies in the hightemperature superconductor $\mathrm{YBa}_{2} \mathrm{Cu}_{3} \mathrm{O}_{7-x}$. Phys. Rev. Mater. 3, 114806 (2019).

23. Magnuson, M. et al. Self-doping processes between planes and chains in the metal-to-superconductor transition of $\mathrm{YBa}_{2} \mathrm{Cu}_{3} \mathrm{O}_{6.9}$. Sci. Rep. 4, 7017 (2014).

24. Sefrioui, Z. et al. Crossover from a three-dimensional to purely twodimensional vortex-glass transition in deoxygenated $\mathrm{YBa}_{2} \mathrm{Cu}_{3} \mathrm{O}_{7-\delta}$ thin films. Phys. Rev. B 60, 15423-15429 (1999).

25. Schwingenschlögl, U. \& Schuster, C. First-Principles calculations of electronic states and self-doping effects at $45^{\circ}$ grain boundary in the high temperature $\mathrm{YBa}_{2} \mathrm{Cu}_{3} \mathrm{O}_{7}$. Phys. Rev. B 102, 22702 (2009).

26. Fujita, K. et al. Spectroscopic imaging scanning tunneling microscopy studies of electronic structuture in the superconducting and pseudogap phases of cuprate high-Tc superconductors. J. Phys. Soc. Jpn. 81, 011005 (2012).

27. Ruan, W. et al. Visualization of the periodic modulation of Cooper pairing in a cuprate superconductor. Nat. Phys. 14, 1178-1182 (2018).

28. Wang, Z. et al. Doping induced mott collapse and possible density wave instabilities in $\left(\mathrm{Sr}_{1-x} \mathrm{La}_{x}\right)_{3} \mathrm{Ir}_{2} \mathrm{O}_{7}$. npj Quantum Mater. 4, 43 (2019).

29. Soumyanarayanan, A. et al. Quantum phase transition from triangular to stripe charge order in $\mathrm{NbSe}_{2}$. Proc. Natl Acad. Sci. USA 110, 1623-1627 (2013).

30. Campi, G. et al. Inhomogeneity of charge-density-wave order and quenched disorder in a high- $T_{c}$ superconductor. Nature 525, 359 (2015).

31. Le Tacon, $\mathrm{M}$. et al. Inelastic $\mathrm{X}$-ray scattering in $\mathrm{YBa}_{2} \mathrm{Cu}_{3} \mathrm{O}_{6.6}$ reveals giant phonon anomalies and elastic central peak due to charge-density-wave formation. Nat. Phys. 10, 52 (2013).

32. Choubey, P., Tu, W.-L., Lee, T.-K. \& Hirschfeld, P. J. Incommensurate charge ordered states in the $\mathrm{t}-\mathrm{t}^{\prime}-\mathrm{J}$ model. N. J. Phys. 19, 013028 (2017).

33. Andersen, N. H. et al. Superstructure formation and the structural phase diagram of $\mathrm{YBa}_{2} \mathrm{Cu}_{3} \mathrm{O}_{6+x}$. Phys. C. 317, 259-269 (1999).
34. Maki, M. Layered charge-density waves with nanoscale coherence in $\mathrm{YBa}_{2} \mathrm{Cu}_{3} \mathrm{O}_{7-\delta}$. Phys. Rev. B 72, 024536 (2005).

35. Ebert, P. et al. Importance of many-body effects in the clustering of charged Zn dopant atoms in GaAs. Phys. Rev. Lett. 83, 757 (1999).

36. Edwards, H. L., Derro, D. J. \& Barr, A. L. Spatially varying energy gap in the $\mathrm{CuO}$ chains of $\mathrm{YBa}_{2} \mathrm{Cu}_{3} \mathrm{O}_{7-x}$ detected by scanning tunneling spectroscopy. Phys. Rev. Lett. 75, 1387-1391 (1995).

37. Maki, M., Nishizaki, T., Shibata, K. \& Kobayashi, N. Electronic structure of the $\mathrm{CuO}$-chain layer in $\mathrm{YBa}_{2} \mathrm{Cu}_{3} \mathrm{O}_{7-\delta}$ studied by scanning tunneling microscopy. Phys. Rev. B 65, 140511 (2002).

38. Maharaj, A. V., Hosur, P. \& Raghu, S. Crisscrossed stripe order from interlayer tunneling in hole-doped cuprates. Phys. Rev. B 90, 125108 (2014).

\section{Acknowledgements}

This work was financially supported by the Ministry of Science and Technology (MOST) of Taiwan (Contract No. MOST 109-2628-M-002-005-MY3, and MOST 1092622-8-002-003), Ministry of Science and Technology (MOST) and German Academic Exchange Service MOST-DAAD Project-Based Personnel Exchange Program (Contract No. 109-2927-I-002-509 and 57447615), National Taiwan Univ.

(Contract No. NTU-109L7848), and the Center of Atomic Initiative for New Materials, National Taiwan Univ., Taipei, Taiwan from the Featured Areas Research Center Program within the framework of the Higher Education Sprout Project by the Ministry of Education in Taiwan (108L9008). B.-C.H. and M.-Y.L. acknowledge the financial support from Academia Sinica through the investigator award (AS-IA-107-M03) granted to Prof. Yuh-Lin Wang. Y.-L.W. and Y.-P.C. are grateful to the budget committee of Institute of Atomic and Molecular Sciences and Institute of Physics, Academia Sinica, respectively for jointly supporting the purchase of the LT-STM used in this study. Thanks to TEM team of Ma-tek for preparing TEM samples and providing high quality TEM images.

\section{Author contributions}

Y.-P.C. designed the project. C.-C.H. and B.-C.H. carried out the experiments. C.-C.H., Y.-L.W., M.-Y.L., and C.-S.C. performed the data analysis and discussed together with M.S., R.E.D.-B., and P.E. the characterization. T.K.L. provided the theoretical understanding. C.-C.H., Y.-P.C., M.S., and P.E. wrote and edited the manuscript. All authors discussed results and commented on the manuscript.

\section{Competing interests}

The authors declare no competing interests.

\section{Additional information}

Supplementary information The online version contains supplementary material available at https://doi.org/10.1038/s41467-021-24003-0.

Correspondence and requests for materials should be addressed to P.E. or Y.-P.C.

Peer review information Nature Communications thanks the anonymous reviewers for their contribution to the peer review of this work.

Reprints and permission information is available at http://www.nature.com/reprints

Publisher's note Springer Nature remains neutral with regard to jurisdictional claims in published maps and institutional affiliations.

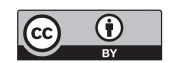

Open Access This article is licensed under a Creative Commons Attribution 4.0 International License, which permits use, sharing, adaptation, distribution and reproduction in any medium or format, as long as you give appropriate credit to the original author(s) and the source, provide a link to the Creative Commons license, and indicate if changes were made. The images or other third party material in this article are included in the article's Creative Commons license, unless indicated otherwise in a credit line to the material. If material is not included in the article's Creative Commons license and your intended use is not permitted by statutory regulation or exceeds the permitted use, you will need to obtain permission directly from the copyright holder. To view a copy of this license, visit http://creativecommons.org/ licenses/by/4.0/.

(c) The Author(s) 2021 